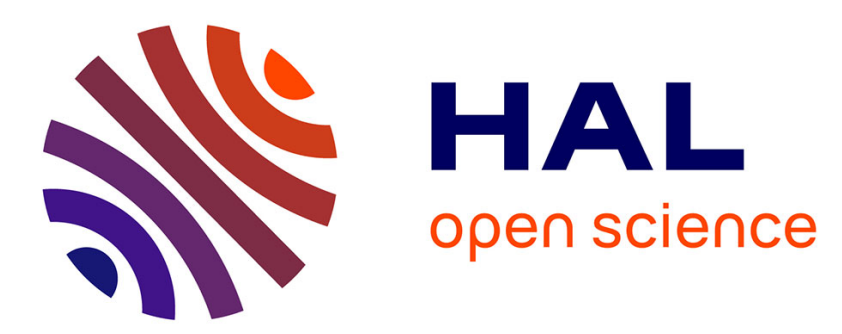

\title{
Un des plus anciens témoignages du dialecte picard? Le cas d'un jugement carolingien (Compiègne, 861)

\author{
Rémy Verdo
}

\section{To cite this version:}

Rémy Verdo. Un des plus anciens témoignages du dialecte picard? Le cas d'un jugement carolingien (Compiègne, 861). Actes del 26é Congrés de Lingüística i Filologia Romàniques (València, 6-11 de setembre de 2010), Sep 2010, València, Espagne. p. 625-638. hal-01769957

\section{HAL Id: hal-01769957 \\ https://hal.science/hal-01769957}

Submitted on 8 Nov 2020

HAL is a multi-disciplinary open access archive for the deposit and dissemination of scientific research documents, whether they are published or not. The documents may come from teaching and research institutions in France or abroad, or from public or private research centers.
L'archive ouverte pluridisciplinaire HAL, est destinée au dépôt et à la diffusion de documents scientifiques de niveau recherche, publiés ou non, émanant des établissements d'enseignement et de recherche français ou étrangers, des laboratoires publics ou privés. 


\section{XXVIé Congrés Internacional de Lingüística i de Filologia Romàniques}

València, 6-11 de setembre de 2010

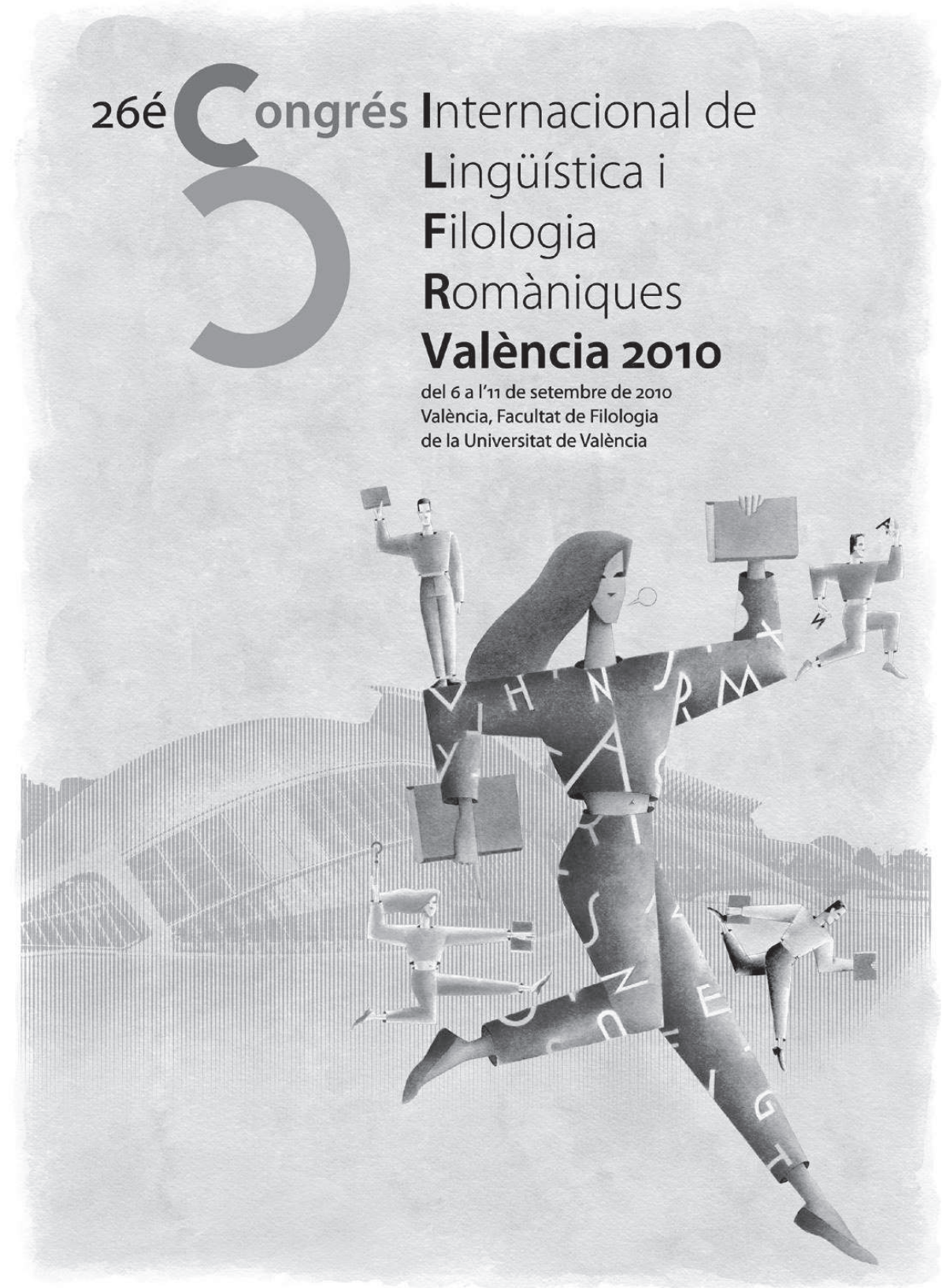



Rémy Verdo (École nationale des chartes)

\section{Un des plus anciens témoignages du dialecte picard? Le cas d'un jugement carolingien (Compiègne, 861)}

L'on dispose de très peu d'études sur la langue des chartes produites en territoire franc après le règne de Charlemagne: pendant longtemps, l'idée a prévalu que la réforme carolingienne a épuré, corrigé la langue, pour lui rendre l'aspect classique qu'elle méritait. ${ }^{1}$ Cette impression appelle au moins de fortes nuances. C'est d'ailleurs ce qui rend l'étude des chartes plus instructive qu'on ne l'a souvent cru: le texte présenté ici devrait le montrer.'

Il s'agit d'un jugement par lequel Charles le Chauve, assisté de huit uassi dominici, de Gailenus, de Fulco, comte du Palais, et de plusieurs autres fidèles, rejette la demande portée devant le tribunal royal par des hommes de Saint-Denis habitant la uilla de Mitry, qui, se prétendant colons libres de naissance, protestaient contre le service servile qu'exigeaient d'eux le maire de Mitry et le moine Deodatus, au ministerium duquel ressortissait ladite uilla.

En s'appuyant sur une typologie assez rigoureuse, on peut assez facilement dégager des phrases entières qui sont en fait de la langue romane habillée en graphie latine. Une fois établie la nature romane de ce texte, il a paru légitime de s'interroger sur la signification des fautes d'orthographe qui donnent un aspect tout à fait particulier à ce texte par rapport à d'autres chartes de structure romane: c'est ce qui laisse à penser que ce texte revêt un intérêt particulier pour les dialectologues. ${ }^{3}$

Sigles utilisés:

LT2 latin tardif d'époque $2\left(\mathrm{VI}^{\mathrm{e}}-\mathrm{VII}{ }^{\mathrm{e}} \mathrm{s}\right.$.)

AFC ancien français classique $\left(\mathrm{XII}^{\mathrm{e}}-\mathrm{XIII}{ }^{\mathrm{e}} \mathrm{s}\right.$.

FEW Franzözisches etymologisches Wörterbuch

(1) In nomine sanctae et indiuidue Trinitatis, Karolus, gracia Dei, rex.

(2) Cum nos in Dei nomine Cumpendio, palacio nostro, SECUs fluuium Isa[ram] ad uniuersorem causas audiendas et recta iudicia TERMINANDUM RESEDEREMUS, ibiQUE uenerunt homines sancti Dionisii EX uilla Mintriaco de ministerium Deodado ${ }^{4}$ monhahyco his nominibus: Ghausselmus, Gotilda cum infantibus suis, Teutlinda, Frodolinda cum infantibus suis uel earum, Siclefrida CUM infantibus suis, Leutfrid $u s$, Teodeuin $u s$, Teuthard $u s$, Teodeilda CUM

1 Je me permets de renvoyer, pour un état général de la question, au travail dont le présent article constitue un extrait remanié (Verdo 2010: 93-98).

2 La transcription qui en est donnée ici reprend l'excellente édition de Tessier (1955: $\left.{ }^{\circ} 314\right)$. On trouvera un facsimilé de l'original dans Lot / Lauer / Tessier (1936: pl. XXXVIII, $\mathrm{n}^{\text {os }} 73$ et $73^{\text {bis }}$ ).

3 L'étude de ce texte a bénéficié en particulier des conseils avisés de Françoise Vielliard et du regretté Jacques Chaurand: qu'ils en soient remerciés chaleureusement.

4 Corrigé sur Deodato. 
infantibus suis, Teutmarus, Teutfrid $u s$, Teutfrida, Bernard $u s$, Bernegarius, Bernehard $u s$, Grimbald us notarius, Bernehilda, Grimhilda, Adalricus, Maurellus, Osanna, Amalricus, Angheuertus, Ursboldus, Grimma cum infantibus suis, Godelfrida CUM infantibus suis, Haistulfus, Hairhardus, Saruinus, Hadebertus, Leutgarius, Rotgarius, Ansoilda, Lurduinus СUM infantibus suis, Hildeberga сUм infantibus suis, Gislinda сuм infandibus, Haldeuerga, Christiana, Raginard $u s$, Siluan $u s$, Odelinda CuM infantibus suis.

(3) Proclamauerunt Se dixerunt eo quod ipsi ex nascendi liberi coloni esse debent sicut alii COLONI sancti Dionisii, et PREdictus Deodadus monachus eis per UIM in INFERIOREM seruicium inclinare uel adfligere uellit INIUSTE.

(4) Tunc interrogauit Fulco comis palaciis et Gailenus memoratum Deodado uel illum maiorem de iam dicta uilla nomine [Antre]ueos qui contra ista familia dicere uel RESPONSARE uellebant.

(5) TUNC in illorum responsum dixerunt quod de presente tales TESTES idoneis COLONIS de PREdicta uilla Mintriaco abebant, per que $m$ eis probare potebant que $m$, in tempore AUI et GENITORI nostri bone memorie Hludouuici, ipsi et illorum antecessores SUPRASCRIPTI serui ad IMFRERIOREM seruicium de iam dicta uilla serper fuissent et plus per drictum et per legem que $m$ COLONI, SICUT manifestum est, fecissent.

(6) Hec sunt nomina TESTIUM qui hoc TESTIFICAUERUNT et de presente supra sanctas reliquias adfirmauerunt: Pascarius, Fulbertus, Acleuertus, Arirhard $u s$, Christoinus, Uuinedulfus, item Pascarius, Matalbertus, Adalricus, filio Bardoni, Tedolgarius, Aghardus, Hildegernus, Flotegarius, Uualfred $u s$, Uuandrehard $u s$, Gislulfus, Uuinehard $u s$, Berdegarius, Godeuert $u s$, Agustin $u$, Bertramn $u s$, Farulfus.

(7) Proinde nos una Cum fidelibus nostris Uuido, Odbertus, Hugo, Bauo, Gerardus, Eurebertus, Alcarius, Hubaldus, uassi dominici, SEU Gailenus et Fulco comis palaciis et alii PLURIMI uisi fuimus iudicasse UT memoratus marior nomine Antreueus in antea adtetisset et UNUMQUISQUE de SEPEdictus seruus ipsum seruicium INFERIOREM, unde de legibus probatus abet, malasset uel repetisset, et ipsum seruicium emendassent et reuadiassent, sIcuT et fecerunt.

(8) Propterea, dum ac causa sit acta uel perpetrata uel legibus DEFINITA esse cognouimus, UT memoratum Deodado monacum UNA CUM [Antre]ueo maiorem partibus sancti Dionisii talem NOTICIA recipere IUXIMUS, per quem SUPRADICTIS seruis ad ipsum seruicium $\underline{\text { in antea }}$ teneant ADQUE elidicant et sit inter eis in POSTMODUM EX ac re sopita et DEFINITA ADQUE INCONUULSA CAUSACIO.

(9) [Actum] Cumpendio palacio supra fuuium Isera. Data I kalendas iulii, anno XxII regnante domno nostro hac gloriosissimo Karolus, rex. In Dei nomine FeLICITER. Amen.

(10) Et Ut hec cercius credatis ADQUE melius conseruetur, de anullo nostro sigilare IUXIMUS. Anscharius notarius scripsit.

N.B.: l'analyse qui suit a dû se restreindre au cœur proprement linguistique de l'étude. ${ }^{5}$

5 Une première partie, intitulée «Situation sociolinguistique: un texte produit par et pour les élites laïques», ainsi qu'une cinquième, intitulée «Réflexions sur la communication romane en Neustrie au milieu du IX ${ }^{\mathrm{e}}$ siècle», s'adressaient plus particulièrement aux historiens. Faute de place, on renverra le lecteur aux pages correspondantes de Verdo (2010: 254-255 et 266-269). 


\section{Un lexique majoritairement commun au latin et au roman}

\subsection{Le texte est présenté ici avec quatre types de précisions}

- Sont figurés en petites capitales les éléments du lexique et de la morphologie verbale tournés vers l'amont de la langue, non attestés en AFC.

- Sont soulignés les éléments lexicaux de création récente, tournés vers l'aval de la langue, qui se maintiennent en AFC.

- Figurent en caractères romains normaux les éléments lexicaux transdiachroniques (non marqués), d'existence ancienne, qui se maintiennent en AFC.

- Apparaissent en italique les abréviations développées, précision nécessaire pour éviter les erreurs de jugement sur certaines graphies.

Cette répartition ne concerne qu'un aspect parmi d'autres; il faudrait par exemple marquer aussi les tournures syntaxiques. Schématiquement, elle vise à traduire la réalité synchronique d'un texte à travers ses éléments diachroniques. Le texte comprend 432 mots; grossièrement, on peut calculer qu'il comporte 10 mots innovants (2,3\%), 66 mots archaïsants $(15,2 \%), 356$ mots transdiachroniques (82,5\%), soit 366 mots qui survivent en ancien français (85\%).

\subsection{Romanismes lexicaux}

1.2.1. L'on retiendra comme annonciateurs de l'aval roman, outre les nouveaux paradigmes verbaux:

- in antea $(\S 7)>\mathrm{AFC}$ 〈enanz〉, c'est-à-dire 〈dorénavant〉;

- de presente $(\S 5)$ : d'après les deux occurrences trouvées dans les bases Brepolis, cette locution adverbiale semble bien avoir été créée en LT2;

- in illorum responsum (§5) / illorum antecessores ( $\$ 5)$ : illorum, par sa morphologie et sa place, est le prototype direct du possessif 〈leur〉 (〈leurs ancêtres〉) et du pronom au cas régime (〈en leur réponse $\rangle=\left\langle\right.$ en réponse à eux〉), en $\mathrm{AFC} ;{ }^{6}$

- et plus per drictum et per legem quem... (§ 5): drictum est un mot de création récente qui supplée le latin rectum, et représente une étape intermédiaire (je n'ai pu dater les changements) entre sa forme latine originale directum et l'AFC «dreit〉. Par ailleurs, dans cet emploi, plus remplace dans les langues romanes l'adverbe magis (magis... quam devient ici plus... quem > AFC 〈plus... que / ke»: «et plus par lui que par compaingnie k’il eüst $\left.\rangle^{7}\right)$. La formule est répandue dans les chartes. ${ }^{8}$

6 Dans les chartes du Ponthieu, lor devient leur à partir de l'année 1283, d'après les relevés de Raynaud (1876: 25).

7 Roman de Tristan en prose, sur http://www.classiques-garnier.com.

8 Cf. «par loi et par droit», dans Monfrin / Fossier / Ruelle (1984: charte n 14 [1246]). 
1.2.2. ... des termes germaniques présents en AFC (isotopes):

- reuadiassent (§ 7): REUUADIARE > anglo-norm. 〈rewager〉 signifiant 〈s'atténuer〉 (cette occurrence en AFC fait figure d'hapax), mais le sens ici est «s'acquitter〉;

- mallasset (\$7): germ. *MAHL (〈lieu du tribunal〉) > latin tardif d'époque 2 mallare $>$ AFC 〈maler〉, c'est-à-dire 〈fixer le jour pour le combat judiciaire〉 (d'après le $F E W$, 〈mal〉 signifiant 〈audience judiciaire〉 est encore attesté en 1616).

2. Une syntaxe et une morphologie majoritairement communes au latin et au roman, à l'exception des formules liminaires

\subsection{Romanismes dans le système verbal}

\subsubsection{Liés à la morphologie}

- potebant $(\S 5)$ : émergence à l'écrit du paradigme innovant de posse > potere, indice que ce texte est conçu en roman.

- uellit (§ 3): paradigme innovant de uelle refait en uellere ${ }^{9}$, où uult est refait en uellèt (et sa variante uellitt, par confusion du $\bar{e}$ et du $\breve{l}$ ). De même, uellebant (§ 4) pour uolebant.

\subsubsection{Liés à l'emploi des temps}

- dixerunt eo quod ipsi ex nascendi liberi coloni esse debent... et predictus Deodatus uellit... (§ 3). Il y a là une entorse à la concordance des temps, commune en $\mathrm{AFC}^{10}$ (où elle peut s'associer à une dissymétrie): «Li compaingnon respondirent que or sont il lié puis qu'il estoient ocis». ${ }^{11} \mathrm{Il}$ s'agit dans ces deux cas d'un discours rapporté. Autre cas plus loin dans le texte (§ 7): probatus abet («qu'il avait prouvé avoir de droit)), au lieu de probatus habebat, où le paradigme de l'infectum devient analytique, au lieu des formes synthétiques probauit ou probauerat. Reste que l'ordre des mots, dans ce syntagme verbal, est traditionnel, car très latinisant, puisqu'en AFC l'on aurait, en langue spontanée, une antéposition des morphèmes suffixés, avec une structure \{auxiliaire + participe passé\}, qui donnerait la tournure 〈a provet〉. En AFC littéraire, on trouve certes l'ordre \{participe + verbe $\}$ du type $\langle\text { provet a }\rangle^{12}$, mais cet ordre, d'ailleurs fréquent avec l'auxiliaire être ${ }^{13}$, est pour lors manifestement, aux $\mathrm{XII}^{\mathrm{e}}-\mathrm{XIII}{ }^{\mathrm{e}}$ siècles, un ordre marqué, donc peu naturel, car lié à des effets de style. Dans le cas des chartes en langue picarde publiées dans la collection Documents linguistiques de la Belgique romane

9 Cf. Haag (1899: 895).

10 Cf. Hasenohr (1993: 180): «l'imparfait de concordance est loin d'être systématique dans les propositions subordonnées».

11 Roman de Tristan en prose, sur http://www.classiques-garnier.com.

12 On en trouve des exemples dans Foulet (1963: 100-105).

13 Anglade (1931: 265). Une étude sur les circonstances de l'emploi d'un tel ordre, en fonction de la chronologie et de l'auxiliaire employé, serait la bienvenue. 
(Monfrin / Fossier / Ruelle 1984-1987), cet ordre apparait dans des morceaux de formules latines transposées telles quelles en graphie française:

* «ces eskievins ki dit sont» (t. 1 [Hainaut], $\mathrm{n}^{\circ}$ 1, 1236/37) // qui dicti sunt;

* «le maison de piere ki nomee est» (ibid.) // quae nominata est.

2.1.3. Liés à la morphologie conjointement à l'emploi des modes et des temps

Le texte ne comporte aucun subjonctif imparfait latin, sauf resederemus $(\S 2)$, qui appartient au formulaire très strict du préambule, présent dans presque tous les jugements.

On a au passé:

- des indicatifs imparfaits, outre potebant (§ 5) et uellebant (§ 4): abebant (§ 5);

- puis des verbes au plus-que-parfait du subjonctif: fuissent (§ 5) et fecissent (§5) sont employés avec leur sens traditionnel de plus-que-parfait. En revanche, le glissement roman du paradigme morphologique est net, puisque les autres plus-que-parfaits du subjonctif ont la valeur de l'imparfait du subjonctif de l'AFC, dont ils sont des prototypes: adtetisset (= adstitisset), malasset, repetisset, emendassent et reuadiassent (tous dans le § 7);

- des parfaits, ancêtres directs des passés simples français: uenerunt (§ 2; > AFC «vin(d) rent), dixerunt (§ 3), interrogauit (§ 4), adfirmauerunt (§ 6), uisi fuimus (§ 7; passif surcomposé très répandu en latin tardif $>\mathrm{AFC}$ (fumes veüs), fecerunt (§ 7), cognouimus $(\S 8)$, scripsit ( $(9)$; restent les verbes proclamauerunt $(\S 3)^{14}$, testificauerunt $(\S 6)$ et iuximus $(\S 8)$, qui ne se sont pas maintenus en $\mathrm{AFC}$;

- à côté desquels apparaît le passé composé: probatus abet (§ 7) avec, comme pour abebant, un romanisme graphique révélateur du $h$ muet.

\subsubsection{Une interrogative indirecte}

qui (= quid) contra ista familia dicere uel responsare uellebant (§ 4): contrairement aux principes du purisme traditionnel, l'interrogative indirecte est à l'indicatif, comme elle l'est en AFC dans la majorité des $\operatorname{cas}^{15}$, et en latin tardif souvent, sous l'influence du grec biblique.

\subsection{Disparition du complément de moyen aprépositionnel}

- de anullo nostro sigilare iuximus (§9).

- per quem eis probare potebant (§5)/ per drictum et per legem (§5), où l'emploi de la tournure prépositionnelle à la place de l'ablatif est une tendance consacrée en AFC.

N.B.: dans per uim (§ 3), l'emploi de per ne doit pas laisser croire qu'il s'agit d'un romanisme, car le diasystème du latin classique emploie déjà per uim concurremment à ui, mot trop court, cf. Gaffiot (s. u. vis) qui cite César (B.G. 1, 14, 3). Il ne s'agit donc pas d'une tournure proprement romane (per + acc. pour remplacer l'abl.) employée avec un vocabulaire classique, uim, qui est éliminé par FORCIA > 〈force〉; mais l'emploi de uim à époque tardive

14 Même si «clamer`se maintient.

15 Cf., pour les nuances qui impliquent le subjonctif, Hasenohr (1993: 192-193). 
est confirmé (surtout avec le sens de 〈violence〉) par les Gloses de Reichenau ${ }^{16}$ (uiolenter est traduit 〈per uim $\rangle$ dans la glose $\mathrm{n}^{\circ} 775$ ).

\subsection{Changement de déclinaison}

Genitori (§5) pour genitoris indique le passage au paradigme de la première déclinaison de type dominus, $-i$. Il est remarquable que ce changement intervienne dans un mot d'emploi archaïque: cela laisse entendre que les mots archaïsants (acrolectaux), voués à être éliminés de la langue naturelle, ont quand même suivi les transformations habituelles.

\subsection{Dissymétries morphologiques et fidélité au formulaire}

- ... regnante domno nostro hac gloriosissimo Karolus, rex (§ 9): le scribe semble avoir suivi le formulaire sans penser à l'adapter, d'où ce début d'ablatif absolu très traditionnel qui se termine par un nominatif (Karolus rex pour Karolo rege), prononcé vraisemblablement [〈regnant dans nostre $\{\mathrm{ac}\}$ gloriosisme Karles, reis〉], où $h a c$ vaudrait pour $a c$.

- nos una cum fidelibus nostris Uuido, Odbertus, Hugo, Bauo, Gerardus, Eurebertus, Alcarius, Hubaldus, uassi dominici, seu Gailenus et Fulco comis palaciis et alii plurimi... (§ 7): la succession d'ablatifs attendue s'interrompt brusquement après fidelibus nostris, au moment où la rédaction s'émancipe du formulaire. Cela semble indiquer que, dans l'esprit du scribe, les morphèmes suffixés de l'ablatif n'ont aucune valeur sémantique et sont omis inconsciemment sitôt qu'il n'y a plus de formulaire à suivre étroitement.

\subsection{La question des réduplications}

Traditionnelles, pour ne pas dire caractéristiques de la langue diplomatique ${ }^{17}$, les réduplications sont nombreuses dans cette charte. Leur fréquence tient probablement à la nécessité de ne laisser aucune échappatoire juridique..$^{18} \mathrm{Il}$ n'est pas non plus impossible qu'elles entrent dans le jeu pragmatique: l'on remarque en effet une tendance à employer des couples de mots de sens parfois très proches, mais de fortunes souvent différentes, dans la mesure où, si l'un des deux termes appartient à la latinité traditionnelle voire franchement archaïque, l'autre a de fortes chances d'appartenir à une latinité plus proche de l'oralité quotidienne (Politzer 1961). Dans le texte, on remarque:

- proclamauerunt se [= seo] dixerunt (§3): le premier terme est réintroduit seulement en moyen français, tandis que le deuxième est, selon le $F E W$, parfaitement commun en AFC...;

- dicere uel responsare ( $\$ 4)$ : le deuxième terme ne figure pas en $\mathrm{AFC}$;

- malasset uel repetisset, et ipsum seruicium emendassent et reuadiassent (§ 7): malasset et reuadiassent sont des mots issus de racines germaniques apparus en latin tardif d'époque 2, et, comme on l'a vu, présents en AFC.

16 Klein / Raupach (1968-1972).

17 Et ce depuis l'Antiquité tardive: cf. Dilcher (1961) et Matzinger-Pfister (1972).

18 C'est un des types de tautologies dégagés par Zimmermann (2003: 222-224). 
L'on doit admettre qu'inclinare uel adfligere (§ 3), comme aui et genitori (§5), et per drictum et per legem ( $(5)$, ne permettent pas de telles suppositions.

Il est donc aisé de caractériser les particularités romanes de ce texte. Mais dans quelle mesure peut-on les localiser précisément?

3. Une graphie latinisante, qui révèle une prononciation largement romane, mais avec des traits picards?

3.1. Un certain nombre d'incongruités apparentes dans la graphie s'expliquent par l'inadéquation entre la réalité phonétique et une tradition graphique caduque

- qui contra... (§ 4) pour quid contra ...,plus... quem (§ 5) pour plus... quam, car quem et quam sont prononcés [kę], quid peut-être prononcé [ki] par analogie avec le pronom masculin qui.

- de sepedictus seruus ( $(7 ;=$ de sepedictis seruis): le $i$ et le $u$ finals atones se confondent, mais la prononciation du $-s$ se maintient en AFC (sepedictus se maintient dans la formule 〈sovent dit(e)〉 courante dans les chartes ${ }^{19}$, même si l'AFC 〈sovent〉 est issu du latin subinde), comme dans tales testes idoneis colonis ( $\$ 5 ;=i d o n e o s$ colonos), le $o$ final n'étant plus qu'une voyelle au timbre indéterminé.

- de ministerium Deodado monhahyco $(\S 2 ;=$ de ministerio Deodati monachi): emploi du datif de possession, tournure latine évitée par les auteurs puristes, qui concurrence très tôt le génitif avant d'être elle-même concurrencée par $a d+$ acc. à partir du vi ${ }^{e}$ siècle (Ernout / Thomas 1964: 73-74). Le reste est commenté plus bas.

- - $m$ final est muet depuis l'Antiquité, ou bien, tout au mieux, la voyelle précédente est nasalisée: ${ }^{20}$ noticia $(\S 8)$ pour noticiam, quem (§ 5) tour à tour pour quos, quod et quam; de ministerium ( $(2)$ est une hypercorrection pour de ministerio, qui se prononçait de la même façon.

- sit pour sic (§ 8): SIC > 〈si > en AFC, le -t final est une tentative de graphie traditionnelle (on se souvenait vaguement que la finale était ce que l'on appelle aujourd'hui une occlusive sourde) en contradiction avec l'oralité. Ce phénomène graphique, conséquence de l'amuïssement des sourdes finales, est fréquent. ${ }^{21}$

- uniuersorem (§ 2): il est vrai que le $o$ initial et contrefinal latin donne, en picard, un $e$ (par exemple: ‘eskemenié` pour 〈excommunié`, cf. Gossen 1970: 91). Mais il s'agit ici d'un $e$ final, qui pourrait s'expliquer par l'influence probable de l'analogie avec l'accusatif en -em attendu après $a d$. On serait ainsi tenté d'y voir une influence des substantifs en -or, -oris.

19 Par exemple dans la charte $n^{\circ} 15$ (datée de 1247) éditée dans Monfrin / Fossier / Ruelle (1984: 25).

20 Exemples eux-mêmes donnés par Rice (1902: 75). Il serait intéressant de dégager la provenance géographique des graphies qui transforment le $-m$ final en $-n$ et celles qui omettent toute forme de nasale.

21 Rice (1902: 58). De quelle époque ce phénomène date-t-il? 
Cette forme, dans tous les cas, n'appartient pas à l'oralité, car en AFC les terminaisons en -orum se maintiennent, sporadiquement, en -or (la 〈gent païenor〉< gentem paganorum). Il semble donc que le scribe ait voulu archaïser l'oralité pour la mettre par écrit, mais se soit trompé dans la reconstruction du morphème suffixé -órum dont la voyelle finale post-tonique était devenue un son indistinct (cf. infra le latin VENĚRUNT > AFC 〈vín(d)rent)).

- ex nascendi ( $\$ 3$; on attendrait ex nascendo): il s'agit probablement d'une substantivation innovante de l'infinitif (e nascere), mais mal archaïsée. ${ }^{22}$ Le texte a été corrigé en et nascendi liberi dans le cartulaire blanc de Saint-Denis ${ }^{23}$, ce qui change le sens de liberi, qui ne signifierait dans ce cas non plus 〈libres〉 mais 〈enfants〉 (nascendi: à naître). Cette leçon est probablement erronée, car 〈enfants〉 se dit bien dans le texte infantes, avec probablement la perte du sens premier de 〈nourrisson〉.

- comis palaciis ( $\$ 4)$, dont on compte deux occurrences, apparaît dans d'autres documents avec comitibus palatiis. C'est une expression qui forme un bloc lexical (une lexie), comme maiordomus, et reçoit de ce fait un $-s$ final.

- infandibus (§ 2): s'agit-il d'une prononciation germanique des sourdes transformées en sonores? ${ }^{24}$ L'hypothèse ne s'accorde guère avec le reste. Beaucoup plus probablement, il s'agit d'un phénomène de sonorisation de la dentale à l'intervocalique, étape intermédiaire avant la chute de cette dernière en AFC (VITA $>*_{\text {VIDA }}>$ vie; FATA $>*_{\text {FADA }}>$ fée) ${ }^{25}$ Le phénomène tend à être plus rare si la voyelle précédant la dentale est une nasale, comme $\mathrm{ici}^{26}$, ce qui rend ce témoignage d'autant plus précieux, si on admet l'interprétation qui en est proposée. ${ }^{27}$ Celle de Carl C. Rice, qui voit dans ces graphies où le $d$ remplace le $t$, non pas un reflet de la prononciation naturelle, mais une simple conséquence de l'ignorance de l'étymologie ${ }^{28}$,

22 Les formulations du type dormire meum, fréquemment utilisées avec une préposition ou un pronom possessif (pro nostro accendere), attestées à l'époque antique, se multiplient à l'époque médiévale, cf. Stotz (1998: 409-410). Le français n'a pas attendu la Renaissance et les emprunts à l'italien pour employer de telles tournures, comme l'atteste par exemple «le terme de lor enfanter» («le jour où elles devaient accoucher»), cf. Hasenohr (1993: 162-163).

23 Cartulaire blanc de Saint-Denis, t. I, p. 470b-471b, n XXVI, «Preceptum Karoli regis quod accepit Deodatus thesaurarius contra mancipia de Mintriaco qui servicium facere recusabant».

${ }^{24}$ Cf. Bresslau (1915: 339), pour la prononciation germanique dans la graphie des diplômes carolingiens de Saint-Gall, territoire passé depuis peu en zone germanophone (cf. Wartmann (1863). Cf. aussi Rice (1902: 58-61).

$25 \mathrm{Si}$, dans enfanz $\left({ }^{*}\right.$ INFANS $<*_{\text {INFAND- } S}<*_{\text {INFANDES }}<$ INFANTES $)$, un phonème dental subsiste dans le $z$ final (prononcé *enfandz), c'est par une sorte d'épenthèse qui se développe entre la nasale $n$ et la sifflante $s$ (et non pas seulement, semble-t-il, «derrière un $n$ qui tombe», comme l'écrit Bourciez 1958: 158).

26 Rice (1902) ne donne aucun exemple de la sorte.

27 Jacques Chaurand m'a écrit à ce sujet: «Considérer la dentale comme intervocalique revient à dire que la nasalisation a fait jusqu'au bout son œuvre. Les phonéticiens datent seulement du $\mathrm{x}^{\mathrm{e}}$ siècle la nasalisation de $a$, mais on peut leur opposer cette sonorisation. L'AFC a enfanz, où $z=t s$, mais la position finale suffit pour expliquer le réassourdissement.»

28 Rice (1902: 61): «The forms quoted from the seventh century leave doubt as to how generally $t$ and $d$ were confused in the intervocalic position. There can be no doubt, however, that in the eighth century $t$ was pronounced without voice and sharply distinguished from $d$, as is shown by spellings like memorathus, vidittur. The frequent occurrence of pedere, marcadum, elidigare in this period simply indicates that the etymology of these words was not yet established. The occurrence of intervocalic $d$ for $t$ in the Cluny documents as late as the eleventh century is to be explained on a 
paraît à peine admissible, le voisement du $t$ intervocalique étant généralement daté bien plus tôt par les phonéticiens..$^{29}$ C'est bien ici un lapsus calami, car il y a dans le texte nombre d'occurrences régulières (infantibus). Au début du texte, le nom Deodatus, volontairement refait en Deodadus, semble témoigner de la plus grande facilité, pour le scribe, d'admettre une graphie plus fidèle à la prononciation lorsqu'il s'agit de noms propres.

- elidicant ( $(8)$, au lieu du traditionnel elitigant, appelle deux remarques: d'une part, le $t$ intervocalique se sonorise en $d$, comme dans infantibus/infandibus; d'autre part, le $g$ transformé en $c$, phénomène qui n'a rien de naturel, représente au contraire probablement une hypercorrection. Le verbe (e)litigare ne survit pas en $\mathrm{AFC}$, et n'a été réintroduit, selon le $F E W$, qu'en moyen français sous la forme 〈litigier». Dans d'autres diplômes, la graphie va même plus loin: ${ }^{30}$ elidiare (comme en espagnol $<e>$ lidiar, selon le $F E W$ ). Il est vrai que cette forme est fréquente dans le formulaire même, cf. Zeumer (1886: 206, 214, 232).

- iuximus (\$ 8): la prononciation de la lettre $x$ à l'intervocalique vaut pour [s], comme en italien (DIXIT $>$ disse), phénomène répandu bien au-delà de l'Italie $;^{31}$ des toponymes comme Auxerre ou Bruxelles l'ont conservé. C'est la prononciation tardo-antique (ce mot n'ayant pas d'avatar dans les langues romanes), que Rice (1902: 88-90) estime avoir été celle pratiquée au centre de la Gaule au moins du VII ${ }^{\mathrm{e}}$ au $\mathrm{IX}^{\mathrm{e}}$ siècle.

- se (§3) pour seo (l'on rencontre cependant un seu à la fin du texte, $\S 7)$, mais il est impossible de voir comment le mot est prononcé dans la scripta picarde où, d'après le $F E W$, il n'existe plus.

- inter eis (\$ 8) pour inter eos, timbre indéterminé dans les monosyllabes, à l'instar des finales.

3.2. La conception de ce texte dans l'aire dialectale du picard, alors en voie d'émergence, permet de s'interroger sur l'apparition de picardismes

- imfreriorem (\$ 5): d'après le $F E W$, l'adjectif inferior n'est pas passé en continu dans l'AFC, mais il a été réintroduit en moyen français. L'on peut formuler deux remarques sur la graphie de ce mot: d'une part, on constate la métathèse du groupe $-e r$ - devenu $-r e^{-32}$, à cette remarque près qu'un $r$ épenthétique (qui remplace l'ancien $r$ changé de place) apparaît après la syllabe, phénomène probablement spontané destiné à éviter le hiatus que donnerait 〈*infreiorem». Une autre hypothèse donnerait INFERIOREM $>$ infreriorem car $f e>$ fre, mais Charles Théodore Gossen $^{33}$ offre assez de cas de dissimilation de $r$ pour invalider cette possibilité. Les exemples

similar basis. Early loan-words like esperit, matire, tradetor show that the voiceless pronunciation of $t$ was preserved throughout the later Merovingian period.»

29 Bourciez (1958: 142-143) place prudemment le voisement de la sourde $t$ en sonore $d$ vers le IV siècle, et, pour la Gaule du nord, le passage du $d$ occlusif au $\delta$ fricatif avant le VIII ${ }^{\mathrm{e}}$ siècle, ce $\delta$ s'effaçant vers la fin $\mathrm{du} \mathrm{XI}{ }^{\mathrm{e}}$ siècle.

30 Rice (1902: 59-60): aelidiatum, elidigatas...

31 Cf., pour le domaine français, Bourciez (1958: 135-136).

32 Phénomène très commun en picard par rapport aux autres parlers de langue d'oïl: cf. Gossen (1970: 153), et, pour ce type de métathèse impliquant le $r$, p. 114: (fremer〉 pour 〈fermer〉, 〈ouvreture) pour 〈ouverture`... la métathèse inverse existe aussi, quoique sensiblement plus rare, comme dans 〈fourment> pour 〈froment».

33 Gossen (1970: 113): 〈cartre〉 et 〈carte〉 (< CARCERE), 〈querrai〉 (〈croirai〉), 〈merkedi〉 (〈mercredi $)$, 〈apenre〉 (〈apprendre〉), etc. 
de $r$ parasite après consonne sont assez fréquents en AFC, notamment «après une consonne intérieure, par suite d'une assimilation progressive» (perdrix < PERDICEM); or, l'assimilation est ici régressive, phénomène que Bourciez ne note que pour les consonnes initiales (trésor < THESAURUM), à moins qu'il ne faille lier la forme qui nous occupe aux nombreux cas d'apparition de la lettre $r$ sans que l'assimilation soit en jeu (rustre < RUSTICUM). ${ }^{34}$

Ce phénomène d'un $r$ épenthétique entre un groupe voyelle $+i o$ peut être rapproché dans cette même charte de la forme marior qui côtoie malgré tout deux occurrences de la forme maiorem: MAIOR > [maiyor] (Bonnard 1975: 33; voir aussi Bourciez 1958: 138). Gossen n'est d'aucun secours dans ce cas précis. D'ailleurs, ce rapprochement reste très contestable, car le $i$ de maior est un $i$ consonne (Bourciez 1958: 138-139). Une latinisation maladroite de 〈maire〉, peut être avancée avec prudence, car 〈marres〉 (pour 〈maires〉, 〈meres〉 ou encore 〈maieur〉) se trouve dans une charte de Flandre. ${ }^{35}$ Une autre hypothèse paraît admissible: on peut se demander si le gaulois maros, (grand), un des composants les plus représentés dans l'anthroponymie gauloise, n'a pas pu parfois être confondu avec maior. La toponymie fournit de nombreux Mareuil (Maro-ialo), mais, dans ce cas, le deuxième composant est gaulois, ce qui rend plus plausible un premier composant de même provenance.

Dans le cas de imfreriorem, reste à remarquer le phénomène bien connu du $n$ transformé en $m$ avant labiale, cf. IN PERPETUUM > imperpetuum, la transformation avant la fricative étant, il est vrai, plus rare mais pas inconnue: on lit 〈Imfers〉 pour 〈Enfer〉 dans le Mistere de Saint Quentin de Jean Molinet, écrit vers $1450^{36}$, et déjà, au milieu du XI ${ }^{\mathrm{e}}$ siècle, le mot 〈emfermetet〉 (< INFIRMitATEM) dans la Vie de saint Alexis (au v. 556). ${ }^{37}$

Le cas d'imfreriorem est d'ailleurs remarquable: étant donné son absence en AFC, si l'on admet qu'il est ici soumis à l'évolution phonétique, c'est qu'il était encore présent dans la romanophonie du $\mathrm{IX}^{\mathrm{e}}$ siècle, ce qui signifierait que les mots en petites capitales sont encore employés иiиа иосе. Par conséquent, leur disparition dans les textes deux siècles plus tard serait le signe que ces mots, déjà anciens dans la langue, étaient socialement marqués comme appartenant à un acrolecte roman, à une parole encore vivante mais de registre stylistiquement élevé car plus proche de l'amont langagier.

- serper ( $(5)$ : aucun rhotacisme du $m$ devant $p$ n’est décrit par Gossen, qui donne

34 Bourciez (1958: 176). - V. Löfstedt (2001: 169-171) qui attribue le $r$ d'un enangelistra trouvé dans deux manuscrits de Sedulius Scott à une analogie avec magistra, car le mot est employé, fait également singulier, au féminin. Il n'en demeure pas moins que Wartburg et Tobler-Lommatzsch, qui attestent la forme 〈evangelistre` en AFC (mais pas au féminin), permettent d'écarter la thèse d'une lectio impossibilis.

35 Monfrin / Fossier / Mantou (1987), n 65 (1270/71): «chis devant dis Ernous ensi com eschevins et Jehan li Wopil ensi com marres».

36 On ne peut prolonger la réflexion faute de trouver d'autres cas similaires chez Gossen (1970).

37 Les bases de données numérisées des éditions Champion, devenues depuis 2009 celles des éditions Garnier (http://www.classiques-garnier.com) permettent de remarquer que la chaîne - $n f$ - est cent fois plus nombreuse que la chaine - $m f$ - (18 884 occurrences contre 187). Les exemples de $m$ devant $f$ semblent proportionnellement plus nombreux chez les auteurs de la zone picarde ou proche, comme en témoigne une recherche comparative des occurrences (parmi les œuvres littéraires des $\mathrm{XI}^{\mathrm{e}}-\mathrm{XIII}^{\mathrm{e}}$ siècles) des chaînes de caractères *nf*/*mf*: Adenet le Roi (né dans le Brabant), Gautier d'Arras et d'autres encore. 
néanmoins, dans un ordre d'idées assez proche, des exemples où le $s$ intérieur devant consonne se transforme en $r$ : AFC $\langle$ vaslet $\rangle=$ picard $\langle$ varlet $\rangle$; de même $\langle$ mesler $\rangle=\langle$ merler $\rangle$; $\langle$ masle $\rangle=\langle$ marle (latin MASCULU[M]); 〈arne〉, 〈arneise (latin ASINU[M]) (Gossen 1970: 107). Le cas est aussi observé pour $l$ : peut-il en être de même pour $m$ ? On sait que le latin semper a donné par métathèse 〈sempre(s)〉 en langue d'oïl: sans pouvoir purement l'affirmer, on est en droit de se demander s'il n'a pas pu parfois donner 〈serpre(s)〉 en picard, à la suite d'une prononciation de la première syllabe analogique à la deuxième. Malheureusement, aucun témoignage n'est disponible pour le prouver. ${ }^{38}$ Cette antéposition du $-r$ - donne une séquence $r+$ consonne $+r$ dont le développement est fréquent en AFC, par épenthèse (PERDICE $>$ 〈perdrix , ORDINE > 〈ordre) ou métathèse (ARBOR > 〈arbre〉, etc.).

Ces cas particuliers, difficiles à analyser, ont en commun la lettre $r$, qui a souvent évolué d'une manière particulière en langue picarde. Enfin, deux exemples paraissent plus probants:

- fuuium (§ 9): Gossen donne incidemment un exemple analogue de chute du $l$ après

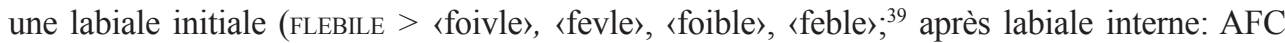
$\langle$ estable $\rangle=$ picard 〈estave (Gossen 1942: 70). À l'inverse, on trouve tout aussi incidemment un $l$ épenthétique dans le paragraphe consacré à l'évolution du suffixe -abulu, -abula, -abile, intercalé entre les deux premières lettres du mot FABULA > 〈faule / 〈f(l)able〉 (Gossen 1970: 111). Ces phénomènes relevés, probablement rares, n'ont pas fait l'objet d'une étude particulière chez Gossen, mais un parallèle avec notre texte est tentant; or, un article de René Debrie consacré au lambdacisme picard attire justement l'attention sur les aspects négligés de la question (Debrie 1985: 167-181) et donne, sans toutefois remonter au-delà du moyen picard, des exemples analogues au nôtre, tels que 〈fanelle〉 pour 〈flanelle〉, 〈fetrir〉 pour 〈flétrir〉, 〈fourin〉 pour 〈florin〉, etc. (Debrie 1985: 177-178).

- monhahyco $(\S 2)=$ monacho. La séquence -nh- est-elle un graphème destiné à marquer la prononciation du $n$ mouillé [ñ] de l'AFC «moingnes? Bourciez (1958: 81) ne donne pas de $n$ mouillé dans ce mot, Gossen n'est pas d'une grande aide sur la question (sauf sur l'équivalence, en picard, de la graphie -gn- / -ngn- pour ce phonème ${ }^{40}$ ), mais l'interrogation des bases Garnier donne une réponse nette. En effet, l'évolution monachus > 〈moingne> n'y apparaît que quatre fois, précisément dans des œuvres picardes ou très proches: Jean Bodel, Raoul de Cambrai, Dou povre mercier ${ }^{41}$ et, plus tardivement et plutôt dans le domaine champenois, Eustache Deschamps (qui a, malgré tout, été bailli de Senlis). L'emploi du $h$ pour marquer la mouillure de la consonne précédente (un $n$ ou un $l$ ), qui semble être caractéristique de l'occitan, n'a pas été consacré par l'ancien picard: on ne le trouve que dans une seule charte des deux volumes des Documents linguistiques de la Belgique romane, dans le mot «conselh〉

38 Ont été consultées sans résultat les bases textuelles informatisées des éditions Garnier et le dictionnaire de Godefroy en mode plein texte.

39 Gossen (1970: 112). - Bonnard (1975: 5) propose aussi pour flebilem, outre l'effet «d'une dissimilation exercée en gallo-roman par le groupe combiné $b l$ qui s'était créé à la seconde syllabe», l'influence de debilem, qui ne vaut pas pour funium.

40 Ainsi que des graphies ign, igni, ingn, et gni relevées par Raynaud (1876: 333).

${ }^{41}$ Il n'y a pas de certitude pour cette œuvre anonyme conservée par un seul manuscrit, car les traits picards y côtoient d'autres traits dialectaux qui font penser à ceux de l'est, cf. Noomen / van den Boogaard (1991: 285-286). 
(Monfrin / Fossier / Ruelle 1984: 91), qui peut aussi bien, semble-t-il, s'écrire 〈consiel〉. ${ }^{42}$ Pour l'AFC, on trouve, hors de la zone picarde, «qui de riens ne l'esparnhe» ${ }^{43}$, seul exemple que nous ayons trouvé dans les bases électroniques des classiques Garnier; pour le moyen français, le résultat est le même: on n'a trouvé que 〈rocinhol〉, chez Eustache Deschamps ${ }^{44}$ déjà cité quelques lignes au-dessus. Le $c$ ne doit plus être qu'une consonne quiescens, peutêtre surajoutée (par pur artifice graphique) au y qui pourrait en être la transcription innovante (prononciation sous forme de yod, où [-náco] $>$ [-niágo] $>$ [-niáyo] $>$ [-gni], comme attesté dans de nombreux toponymes ${ }^{45}$ ).

\section{Bibliographie}

Anglade, Joseph (1931): Grammaire élémentaire de l'ancien français. Paris: A. Colin.

Bonnard, Henri (1975): Synopsis de phonétique historique. Paris: Société d'édition de l'enseignement supérieur.

Bourciez, Édouard (1958): Précis de phonétique française. Paris: Klincksieck.

Bresslau, Harry (1912-1932): Handbuch der Urkundenlehre für Deutschland und Italien. Leipzig: Von Veit \& Comp.

Debrie, René (1985): Réflexion sur le comportement de la liquide 1 en phonétique picarde. In: RLiR 49, 193-194, 167-181.

Dilcher, Gerhard (1961): Paarformeln in der Rechtssprache des frühen Mittelalters. Darmstadt: C. Rinck.

Ernout, Alfred / Thomas, François (1964): Syntaxe latine. Paris: Klincksieck.

Foulet, Lucien (1963): Petite syntaxe de l'ancien français. Paris: Champion.

Gossen, Charles Théodore (1942): Die Pikardie als Sprachlandschaft des Mittelalters, auf Grund der Urkunden. Bienne: Schüler.

- (1970): Grammaire de l'ancien picard. Paris: Klincksieck.

Haag, Oskar (1899): Die Latinität Fredegars. In: RF 10, 5, 835-932.

Hasenohr, Geneviève (1993): Introduction à l'ancien français de Guy Raynaud de Lage. Paris: SEDES.

Klein, Hans-Wilhelm / Raupach, Manfred (1968-1972) (edd.): Die Reichenauer Glossen. Munich: Hueber / Fink.

Löfstedt, Bengt (2001): Die Form evangelistra. In: Archivium latinitatis Medii Aevi (Bulletin Du Cange) 59, 169-171.

Lot, Ferdinand / Lauer, Philippe / Tessier, Georges (edd.) (1936): Diplomata Karolinorum: recueil de reproductions en fac-similé des actes originaux des souverains carolingiens conservés dans les

${ }^{42}$ D'après Raynaud (1876: 30) où l'interversion des lettres $e i>i e$, quoique assez peu répandue, sert à marquer la mouillure, de même que $i l$ («conseil〉), ill («conseill〉), $l$ («consel»), ll («consell〉), et llie devant -ia latin (〈fillie〉), comme le montre plus loin le même auteur (1876: 330-331).

43 Roman de Tristan en prose, sur http://www.classiques-garnier.com.

${ }^{44}$ Cuvres complètes d'Eustache Deschamps, sur http://www.classiques-garnier.com.

45 Bourciez (1958: 44). On pourrait citer la ville de Moigny-sur-École (dans l'Essonne), anciennement Moniacum, ce qui renforce notre idée: pour que monac(h)um donne «moigne`, il fallait que le $n$ fût mouillé. Cela laisserait à penser que, à la différence de ce qu'enseigne Bourciez (1958: 81), monachum n'a pas forcément toujours donné *monı̌cu à l'époque latine tardive dans le nord de la Gaule. 
archives et bibliothèques de France. Toulouse / Paris: Privat / Didier, t. 4.

Matzinger-Pfister, Regula (1972): Paarformel, Synonymik und zweisprachiges Wortpaar: zu mehrgliedrigen Ausdrucksweise der mittelaterlichen Urkundensprache. Zurich: Juris Verlag.

Monfrin, Jacques / Fossier, Lucie / Ruelle, Pierre (edd.) (1984-1987): Documents linguistiques de la Belgique romane. 1: Chartes en langue française antérieures à 1271 conservées dans la province du Hainaut; 2: Chartes en langue française antérieures à 1271 conservées dans les provinces de Flandre orientale et de Flandre occidentale. Paris: CNRS.

Noomen, Willem / van den Boogaard, Nico (1991): Nouveau recueil complet des fabliaux, t. VIII. Assen: Van Gorcum.

Politzer, Robert L. (1961): Synonymic repetition in late Latin and Romance. In: Language: journal of the Linguistic Society of America 37, 4, 484-487.

Raynaud, Gaston (1876): Étude sur le dialecte picard dans le Ponthieu, d'après les chartes des XIII et XIV siècles [premier article]. In: Bibliothèque de l'École des chartes 37, 1, 5-34 et 317-357.

Rice, Carl C. (1902): The phonology of Gallic clerical Latin after the sixth century: an introductory historical study based chiefly on Merovingian and Carolingian spelling and on the forms of old French loan-words. Cambridge: Mass.

Stotz, Peter (1996-2004): Handbuch zur lateinischen Sprache des Mittelalters. Munich: C. H. Beck.

Tessier, Georges (1955): Recueil des actes de Charles II le Chauve, roi de France. Paris: Académie des Inscriptions et Belles-Lettres.

Verdo, Rémy (2010): La reconfiguration du latin mérovingien sous les Carolingiens: étude sociolinguistique des diplômes royaux et des réécritures hagiographiques (VII -IXe siècle), thèse de l'École des chartes. Paris.

Wartmann, Hermann (1863): Urkundenbuch der Abtei St. Gallen, Theil 1 (Jahr 700-840). Zürich.

Zeumer, Karl (éd.) (1886): Formulae Merowingici et Karolini aevi. Hanovre: Hahn.

Zimmermann, Michel (2003): Écrire et lire en Catalogne (IXe-XII siècle). Madrid: Casa de Velázquez. 